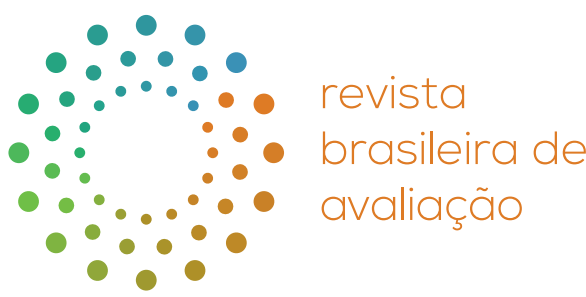

\title{
Fatores para a profissionalização e a importância das diretrizes para a prática da avaliação no Brasil
}

\author{
Factors that influence professionalization and the importance of \\ guidelines for the practice of evaluation in Brazil
}

Márcia Paterno Joppert ${ }^{1,2 *}$ [branca] (D)

${ }^{1}$ Rede Brasileira de Monitoramento e Avaliação, Rio de Janeiro, RJ, Brasil

${ }^{2}$ Claremont Graduate University, Claremont, CA, Estados Unidos da América

COMO CITAR: Joppert, Márcia Paterno. (2021). Fatores para a profissionalização e a importância das diretrizes para a prática da avaliação no Brasil. Revista Brasileira de Avaliação, 10(1), e100321. https://doi.org/10.4322/ rbaval202110003

\section{Resumo}

O desenvolvimento da avaliação no Brasil como campo profissional pode ser observado por diversos aspectos. Mais oportunidades de treinamento e formação e consequente produção acadêmica, tendência de institucionalização da função avaliação nas organizações, aumento da demanda por avaliações externas e o crescimento da Rede Brasileira de Monitoramento e Avaliação (RBMA) são alguns sinais. Este ensaio, elaborado a partir de revisão de literatura, pretende realizar uma reflexão sobre os fatores que levam à profissionalização da avaliação nos diversos países onde se desenvolveu e a importância de se ter diretrizes, princípios, normas e padrões como fator decisivo para seu aprimoramento e relevância. Pretende também refletir sobre o potencial das recém lançadas diretrizes brasileiras para o desenvolvimento do campo no Brasil.

Palavras-chave: Avaliação. Avaliação profissional. Competências em avaliação. Padrões em avaliação. Ética em avaliação. Associações de avaliação.

\section{Abstract}

The development of evaluation in Brazil as a professional field can be observed by several aspects. The growth in the offer of training and education opportunities and the consequent academic production, the institutionalization movements in different organizations, the increase in demand for external evaluations and the growth of the Brazilian Monitoring and Evaluation Network (RBMA) are some signs. This essay, based on a literature review, intends to reflect on the factors that lead to the professionalization of evaluation in the different countries where it was developed and the importance of having guidelines, principles, norms and standards as a decisive factor for its improvement and relevance. It also intends to reflect on the potential of the recently launched Brazilian guidelines for the development of the field in Brazil.

Keywords: Evaluation. Professional evaluation. Competencies in evaluation. Evaluation standards. Ethics in evaluation. Evaluation associations.

Recebido: Outubro 19, 2020 Aceito: Fevereiro 02, 2021

*Autor correspondente: Márcia Paterno Joppert E-mail: marcia.joppert@gmail.com

\section{(c) BY}

Este é um artigo publicado em acesso aberto (Open Access) sob a licença

Creative Commons Attribution, que permite uso, distribuição e reprodução em qualquer meio, sem restrições desde que o trabalho original seja corretamente citado. 


\section{Introdução}

A avaliação de políticas, programas e projetos no Brasil vem ganhando cada vez mais relevância nas últimas décadas. Esta relevância está claramente sinalizada por alguns fatores. As oportunidades de treinamento e formação no Brasil têm crescido com a criação de novos cursos de curta duração, graduação e pós-graduação em monitoramento e avaliação. Também existem algumas iniciativas de institucionalização no setor público, principalmente nas esferas federal e estadual. A criação do Conselho de Monitoramento e Avaliação de Políticas Públicas, de natureza interministerial, por decreto em 2019 (Brasil, 2020), sinaliza uma intenção de avaliar políticas públicas de nível federal. Importante citar também a atuação da Secretaria de Avaliação e Gestão da Informação do Ministério da Cidadania, que, desde sua criação em 2004, realizou estudos, publicação e formação de gestores em monitoramento e avaliação de projetos sociais (Brasil, 2016). Houve ainda um aumento da demanda por avaliações externas. Dados do relatório anual da Rede Brasileira de Monitoramento e Avaliação (RBMA) de 2020 revelaram que nos últimos 5 anos foram divulgadas 222 oportunidades de trabalho, incluindo vagas para compor as equipes internas em instituições e editais de seleção para contratação de consultores individuais ou organizações. Por fim, um último fator é o crescimento do número de adeptos (atualmente mais de 8.500) e das iniciativas da RBMA voltadas à profissionalização, apoiadas por diversos parceiros. Destacam-se a recente publicação das Diretrizes para a Prática da Avaliação no Brasil, em agosto de 2020, e o relançamento da Revista Brasileira de Avaliação (RBMA, 2021a).

Esses fatores criam um ambiente cada vez mais favorável para o desenvolvimento do campo no Brasil e tornam cada vez mais necessário avançar em iniciativas que contribuam para a profissionalização da atividade no país, com a consequente melhoria da sua qualidade e relevância para os tomadores de decisão e para a sociedade. A avaliação, em última instância, é um instrumento de fortalecimento da democracia, por promover a compreensão e visão crítica de como as políticas públicas são formuladas e no que resultam. Esse entendimento é fundamental para embasar as escolhas típicas do exercício democrático.

Avaliações de qualidade podem contribuir para a efetividade, eficiência, eficácia e legitimidade de iniciativas. Uma das características de uma avaliação de qualidade é que seus resultados sejam úteis. Para que isso ocorra, avaliações devem ser realizadas com o envolvimento daqueles que vão utilizar seus resultados, por exemplo, para tomar decisões a respeito das iniciativas (Patton, 2008). Avaliações podem ter diversos usos como ajudar a reformular e implementar iniciativas, tirar conclusões sobre seu valor, disseminar práticas bem-sucedidas e promover esclarecimento junto aos atores envolvidos (Stufflebeam \& Coryn, 2014). Avaliações formativas, realizada quando as iniciativas estão em fase de implementação, têm por principal objetivo contribuir para a melhoria de sua qualidade. Esse tipo de avaliação auxilia os tomadores de decisão a promoverem mudanças positivas nos desenhos dos programas, das políticas e nas próprias organizações empreendedoras. Especialmente quando há envolvimento das comunidades, é possível identificar as razões pelas quais uma política deve ser alterada, com base em suas necessidades ou "[...] nas inequidades em termos de acesso, distribuição ou implementação de tais políticas" (Mertens, 2008, p. 340).

Avaliações somativas, por sua vez, são retrospectivas, ou seja, se aplicam a iniciativas já concluídas e têm como principal resultado realizar um julgamento do valor da iniciativa, de seus alcances. A descontinuidade de uma iniciativa estaria, desse modo, baseada em evidências sobre seu mérito e seu valor. Tanto iniciativas em andamento quanto concluídas, têm em geral, como público-alvo grupos, da sociedade. Portanto, a melhoria no seu desenho ou o julgamento sobre o seu mérito e valor, tem impacto direto sobre a vida de pessoas. Um outro uso é ajudar na disseminação de iniciativas ou produtos que tenham sido considerados bem-sucedidos ou de boa qualidade, orientando consumidores ou beneficiários em suas escolhas. Por fim, um quarto tipo de uso é produzir informações sobre o que funciona ou não. Isso se dá a partir da avaliação um conjunto de iniciativas ao longo de um certo período de tempo, orientando formuladores de políticas. A partir desses diversos tipos de uso, avaliações podem contribuir para uma melhor compreensão de políticas e suas consequências por parte da sociedade, promovendo uma iluminação social (Stockmann, 2016). Stockmann usa 
esse termo para ilustrar essa compreensão, que também tem um efeito direto na vida de pessoas no sentido de aumentar os seus conhecimentos para fazer suas escolhas políticas. Por todo exposto, uma avaliação de qualidade pode fortalecer a democracia. Por isso, o tema foi escolhido para este ensaio.

O objetivo desse ensaio é buscar a compreensão de quais fatores influenciam a qualidade em avaliações e em que medida diretrizes e padrões são especialmente relevantes para uma maior profissionalização dessa atividade. O ensaio também visa discorrer sobre as diretrizes brasileiras, recém lançadas pela RBMA, sua origem, processo de construção e relevância.

A metodologia utilizada foi a revisão de literatura relacionada à profissionalização da avaliação em diversos países, a partir das referências bibliográficas do curso de doutorado em avaliação e métodos de pesquisa aplicados da Claremont Graduate University, do qual a autora é aluna, bem como de informações pesquisadas sobre o movimento global que se criou em torno da avaliação a partir da iniciativa EvalPartners em 2012, no qual a autora tem estado pessoalmente envolvida desde então. A pesquisa bibliográfica foi realizada a partir de buscas por palavras-chave na plataforma Google Scholars, em páginas da internet e no repositório virtual dos Claremont Colleges.

\section{A profissionalização da avaliação}

Segundo Podems (2018) não existe ainda um consenso entre teóricos do campo da avaliação sobre quem pode ser considerado ou não avaliador e se avaliação pode ou não ser considerada uma profissão. Essa questão tem provocado muitos debates e reflexões na comunidade de avaliação, no mundo todo. O Australian Council of Professions define profissão como:

[...] um grupo disciplinado de indivíduos que aderem a padrões éticos e que se apresentam, e são aceitos pelo público, como possuidores de conhecimentos e habilidades especiais em um corpo de aprendizagem amplamente reconhecido derivado de pesquisa, educação e treinamento de alto nível, e que estão preparados para aplicar esse conhecimento e exercer essas habilidades de acordo com interesses de outros. (ACoP, 2021, tradução livre da autora).

Ao aplicar esta definição ao campo da avaliação, poderíamos dizer que avaliadores são um grupo de indivíduos que aderem às normas e diretrizes éticas de avaliação, e que se apresentam e são reconhecidos como possuidores de conhecimentos e habilidades especiais no corpo de aprendizagem derivados de programas de educação e treinamento em avaliação, teorias, competências e pesquisa, e estão preparados para aplicar esses conhecimentos e habilidades no interesse das organizações. O termo avaliadores aqui refere-se aos que exercem os diversos papéis em uma avaliação, incluindo os representados por quem demanda ou contrata estudos, os pesquisadores ou consultores que os conduzem e os que usam seus resultados.

Conforme conceituado por algumas organizações de referência, como a Organização Internacional para Cooperação em Avaliação (IOCE) e o Grupo de Avaliação das Nações Unidas (UNEG), a profissionalização da avaliação é fortemente influenciada por um conjunto de fatores. O documento conceitual do UNEG (2016) propõe uma estrutura de pensamento sobre a profissionalização da avaliação em nível global. Isso ocorre a partir do reconhecimento de que avaliação é essencial para o avanço do desenvolvimento dos países, possibilitando mudanças e que, para que a prática da avaliação seja levada a sério, é preciso que ela se diferencie como uma profissão.

A estrutura proposta considera um conjunto de fatores que contribuem para a profissionalização da avaliação e foi construída como parte da Agenda Global de Avaliação 2016-2020 (EvalPartners, 2018). Esses fatores podem ser articulados e priorizados de diferentes maneiras por organizações que usam avaliações, ou por Organizações Voluntárias para a Profissionalização da Avaliação (VOPEs, sigla em inglês) que promovem a profissionalização nos países. Cada um desses fatores tem se desenvolvido de forma diferente nos diversos países e esse desenvolvimento tem sido observado e registrado em vasta literatura. 
A Figura 1, a seguir, apresenta os seis fatores definidos pela UNEG que contribuem para profissionalização da avaliação:

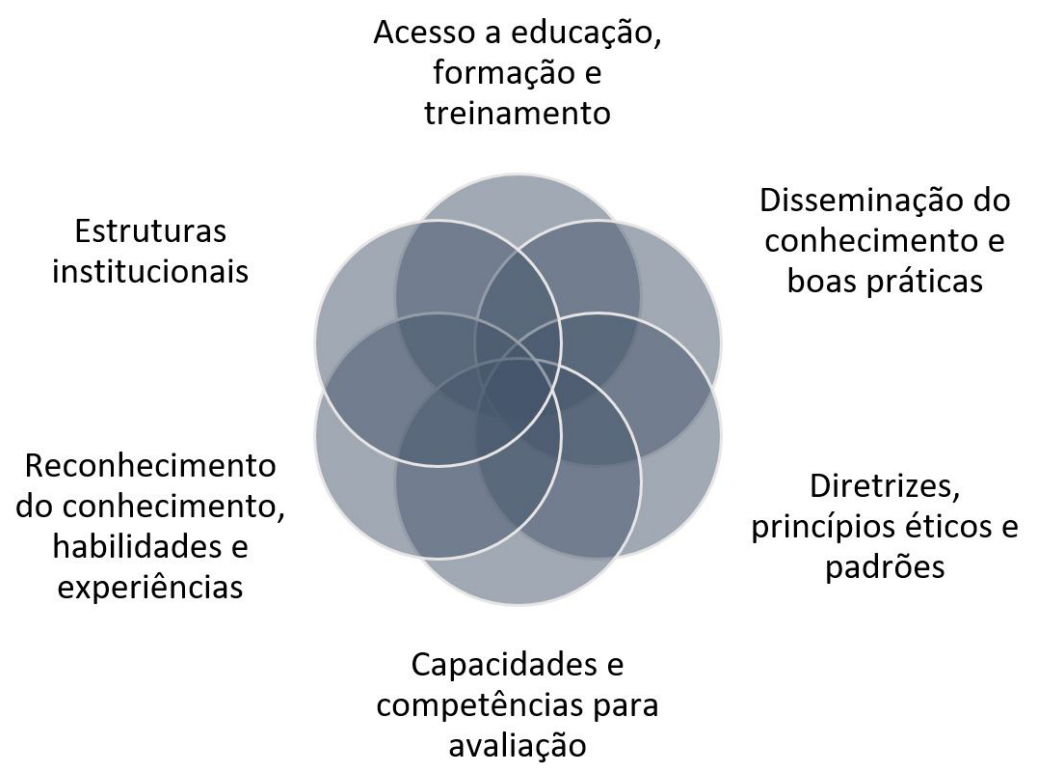

Figura 1. Seis fatores que contribuem para a profissionalização da avaliação.

Fonte: elaboração própria, a partir do documento conceitual da UNEG (2016, p. 7).

Acesso a educação, formação e treinamento

O acesso a educação, formação e treinamento ocorre de diversas formas, desde cursos de curta duração, workshops de desenvolvimento profissional, mentoria e cursos de pósgraduação, muitas vezes disponíveis online. Não há um caminho único.

No Brasil, as oportunidades de educação, formação e treinamento na área de avaliação têm crescido nas duas últimas décadas. Como opções de cursos de curta duração, a Fundação Itaú Social oferece, desde 2004, o Programa de Avaliação Econômica de Projetos Sociais, em níveis básico e avançado (Fundação Itaú Social, 2021). O Centro de Aprendizagem em Avaliação e Resultados (CLEAR) - Brasil e África Lusófona oferece curso de formação em monitoramento e avaliação do para gestores públicos e de programas sociais e potenciais avaliadores (FGV EESP Clear, 2020; FGV, 2020). Há também no Brasil um curso de graduação em gestão da avaliação, oferecido pela Faculdade Cesgranrio (2020c), e algumas ofertas de pós-graduação em avaliação, sendo as mais conhecidas: o mestrado profissional em avaliação da Faculdade Cesgranrio (2021), o mestrado acadêmico em Avaliação de Políticas Públicas da Universidade Federal do Ceará (UFC, 2021) e o mestrado profissional em Gestão e Avaliação da Educação Pública da Universidade Federal de Juiz de Fora (UFJF, 2021). Recentemente, foram criados o programa de mestrado profissional em Avaliação e Monitoramento de Políticas Públicas da Escola Nacional de Administração Pública (ENAP, 2021), voltado a gestores públicos, o Programa de Pós-Graduação em Métodos e Gestão em Avaliação oferecido pela Universidade Federal de Santa Catarina (UFSC, 2021) e o curso de especialização em monitoramento e avaliação de políticas públicas da Fundação João Pinheiro (FJP, 2020). Há também diversas opções já mapeadas pelas VOPEs na América Latina (Rodriguez-Bilella, 2018), Estados Unidos (AEA, 2020c) e Europa (EES, 2020b).

Em países em que um conjunto de capacidades e competências para os avaliadores já foi definido, como o Canadá, há uma tendência em se articular o fator acesso a educação, formação e treinamento e o fator capacidades e competências. Segundo Gauthier et al. (2015), a partir da definição de competências e da criação de um programa de credenciamento em avaliação, diversos cursos de pós-graduação em avaliação foram criados em universidades canadenses, coordenados em um consórcio, com apoio da Sociedade Canadense de Avaliação. 
O Consórcio de Universidades para Educação em Avaliação (CUEE) tem a finalidade de aumentar o acesso à educação em nível de pós-graduação de alto nível que permita a seus graduandos adquirir as competências requeridas para atuar como avaliadores e eventualmente cumprir os requisitos acadêmicos para a sua designação como avaliador credenciado (CE), conferida pela Sociedade Canadense de Avaliação". (CUEE, 2021, tradução livre da autora).

Schwandt (2015) afirma que quando as escolas profissionais são submetidas a processos de acreditação, supõe-se que elas conectem padrões, competências e currículo. No entanto, este tipo de mecanismo não existe no campo da avaliação. Portanto, não é possível ter certeza se a ampla gama de oportunidades de educação e treinamento é suficiente ou adequada para formar profissionais que poderão contratar, conduzir e usar avaliações sólidas e também aqueles que poderão ensinar e conduzir pesquisas na área. Uma revisão da literatura e da prática concluiu que “[...] mais trabalho é necessário para levar a educação do avaliador adiante, de modo que possa atender com mais eficácia as demandas de aprendizagem e desempenho de nosso contexto global" (Gullickson et al., 2019, p. 27). O artigo de Gullickson et al. (2019) também sugere que haja uma lente de avaliação contínua sobre os esforços de educação para melhorar o ensino e as práticas de avaliação.

Segundo Meyer (2016), ainda existe uma relação difícil entre o ensino acadêmico e a prática da avaliação. Enquanto o ensino acadêmico deve seguir os valores, regras e padrões do sistema acadêmico, a avaliação, como ciência aplicada, deve considerar o mercado, as pressões de custo e tempo e ainda a demanda dos clientes que pagam pelos serviços. O amadurecimento do campo da avaliação depende, em certa medida, da articulação dessas duas vertentes, de modo que o ensino contribua para que a prática da avaliação tenha cada vez mais qualidade e relevância e que a prática da avaliação gere conhecimentos que possam enriquecer as atividades de ensino. A pesquisa em avaliação seria o terceiro pilar que ajudaria a estruturar a avaliação como disciplina.

Disseminação de conhecimento e boas práticas

A disseminação do conhecimento e boas práticas em avaliação depende da formulação de teorias e abordagens e do registro da prática e da pesquisa nesse campo. Pode dar-se de diversas maneiras como publicações ou apresentações em conferência. Essa disseminação é fundamental para a formação de um corpo de conhecimento no campo. Nesse sentido, os livros, as revistas especializadas e os seminários promovidos pelas VOPEs no mundo todo, em especial a Associação Americana de Avaliação (AEA), a Sociedade Canadense de Avaliação (CSE) e a Sociedade Europeia de Avaliação (EES) têm representado as principais estratégias.

No Brasil, a RBMA tem, desde 2008, promovido a disseminação de informações e conhecimento por meio de seu portal, da realização de 9 seminários nacionais, workshops, e dos 9 números já publicados da Revista Brasileira de Monitoramento e Avaliação, que está sendo relançada em 2020 sob o nome Revista Brasileira de Avaliação (RBMA, 2021b). Há também outras revistas consagradas no campo como a Revista Avaliação de Políticas Públicas - AVAL, da Universidade Federal do Ceará (UFC, 2020), a Revista Estudos em Avaliação Educacional, da Fundação Carlos Chagas (FCC, 2020), Revista da Avaliação da Educação Superior (SciELO, 2020), a Revista Meta: Avaliação e a Revista Ensaio: Avaliação e Políticas Públicas em Educação (as duas últimas da Faculdade Cesgranrio, 2020a, 2020b). Outras organizações têm promovido eventos voltados à avaliação. O Grupo de Institutos, Fundações e Empresas (GIFE) promove frequentemente seminários internacionais de avaliação. O CLEAR, por sua vez, tem organizado anualmente, desde 2019, a Semana de Avaliação gLOCAL que promoveu 263 eventos online promovidos em 37 países em 6 idiomas, em 2019, e 254 eventos online promovidos em 38 países em 5 idiomas, em 2020.

Outras ferramentas inegavelmente úteis para a troca de experiências e conhecimentos são os mecanismos online que foram criados nos últimos anos, como listas e grupos eletrônicos que vêm sendo criados nas redes sociais. Uma simples pesquisa na plataforma do Linkedln retornou 3.533 grupos que incluem a palavra "avaliação" em seus nomes. 
Diretrizes, princípios éticos e padrões

Conforme a definição do Australian Council of Professions, citada anteriormente neste ensaio, toda profissão deve estar baseada em padrões éticos segundo os quais se espera que os profissionais atuem. A partir do momento em que se percebeu a relação entre a avaliação e a ética, tornou-se importante a elaboração de diretrizes e padrões. A pioneira foi a Associação Americana de Avaliação (AEA), que elaborou os Princípios Orientadores para avaliadores (Guiding Principles for Evaluators) em 1994 (AEA, 2020b). Esse documento, que tem sofrido revisões a cada 5 anos, tornou-se ponto de partida para outras VOPEs e organizações internacionais. O UNEG (2017) lançou, em 2005, as Normas e Padrões para Avaliação e o Comitê de Ajuda ao Desenvolvimento da Organização para a Cooperação e Desenvolvimento Econômico (CAD/OCDE) publicou, em 2010, as Normas do CAD para a Qualidade da Avaliação do Desenvolvimento. A partir desses primeiros movimentos, várias VOPEs elaboraram guias, diretrizes e padrões (OCDE, 2010). Em 2015 foi a vez da Rede de Monitoramento, Avaliação e Sistematização da América Latina e o Caribe (ReLAC) que, apoiada pelo Projeto de Promoção de Capacidades de Avaliação (FOCEVAL), e pelo Instituto Alemão de Avaliação para a Cooperação para o Desenvolvimento (DEval), lançou os Padrões de Avaliação para a América Latina e o Caribe (ReLAC, 2016). Inspirada nos resultados da ReLAC, a RBMA conduziu um processo de envolvimento da comunidade avaliativa brasileira e lançou, em agosto de 2020, as Diretrizes para a Prática da Avaliação no Brasil (Silva et al., 2020), cujo processo e características serão descritos mais adiante neste ensaio.

A elaboração de diretrizes, princípios éticos e padrões é iniciativa em geral empreendida pelas VOPEs ou por organizações cuja institucionalização da avaliação está bastante avançada, como o sistema das Nações Unidas e a OCDE. Segundo Bustelo (2006), dispor de diretrizes e padrões para a prática da avaliação é um importante compasso ético para guiar a prática profissional. Conforme disse Morris (2011), ética pode referir-se a comportamentos morais em geral, princípios de conduta de uma profissão em particular ou ao conjunto de valores, crenças e ações relevantes para a atividade que está sendo conduzida. Todos eles são absolutamente aplicáveis à avaliação.

Como já foi dito, as diretrizes podem contribuir para que a avaliação se torne mais profissional e consiga contribuir efetivamente para a melhoria dos objetos avaliados e das organizações que os promovem. Em particular, as diretrizes podem ter grande importância no apoio aos processos de treinamento e formação de profissionais, por exemplo, os relacionados à prática avaliativa e seus desafios éticos, que podem ser elucidados por casos práticos (Bustelo, 2006). Portanto, todo conhecimento empírico que tem origem nas pesquisas e práticas avaliativas pode ter diretrizes como parâmetros de qualificação.

Diretrizes também são usadas como parâmetros para o desenvolvimento dos processos de credenciamento existentes em algumas VOPEs e, mesmo quando esses processos são inexistentes, contribui, conforme Bustelo (2006), para fomentar o sentimento de pertencimento e identidade entre os profissionais das diversas comunidades de avaliadores nos diferentes países e regiões e fortalecer uma cultura de avaliação. Por fim, estruturas voltadas a institucionalizar a avaliação nas organizações públicas e privadas, bem como as próprias VOPEs, utilizam diretrizes como parâmetro geral de atuação profissional.

Capacidades e competências para avaliação

A definição das capacidades e competências para avaliadores tem também sido empreendida por diversas VOPEs e por organizações internacionais. Um conjunto de competências acordadas pode nortear a definição de perfis de profissionais para assumir posições nas áreas responsáveis por avaliações em organizações ou para definir os perfis desejáveis de avaliadores ou equipes de avaliadores ou ainda, para servir de bússola na formação e atuação profissional dos próprios profissionais.

A partir dos Guiding Principles, a AEA elaborou as Competências dos Avaliadores (AEA, 2020d). Este documento tenta criar uma linguagem comum e um conjunto de critérios para esclarecer o que significa ser incluído na definição de avaliador. Segundo a AEA (2020a), a intenção das competências é tornar claras as características da prática profissional da avaliação e 
desafiar a comunidade a criar caminhos para engajar todos os tipos de pessoas a tornaremse avaliadores.

As reflexões sobre a prática levaram outras VOPEs a definirem um conjunto de competências que podem ser definidas como conjuntos de conhecimentos e habilidades, natas ou aprendidas, e outros requisitos necessários para um desempenho bem-sucedido (UNEG, 2013). Segundo Stevahn et al. (2005, p. 52), as competências para a prática profissional da avaliação "[...] se concentram em normas e valores fundamentais subjacentes à prática de avaliação, como adesão aos padrões de avaliação e ética".

Wilcox \& King (2012) apontaram que o valor do desenvolvimento de competências ainda não está claro para a comunidade de avaliadores e ainda não existem estudos empíricos que mostrem que elas se refletem em avaliações mais úteis e sólidas. As competências do avaliador podem ser usadas como base para programas de treinamento e para desenvolver processos de certificação ou credenciamento. Podem representar ainda um conjunto de critérios para reconhecer quem são os profissionais qualificados e experientes e, consequentemente, dar continuidade ao movimento do campo para fazer da avaliação uma profissão.

Podems (2014) conduziu um estudo multinacional sobre o que estava acontecendo em termos de competências de avaliadores e de que maneira elas estariam influenciando a profissionalização da avaliação. Cinco casos foram selecionados mostrando diferentes contextos e perspectivas: Aotearoa New Zealand Evaluation Association (ANZEA), Canadian Evaluation Society (CES), South African Department of Performance Monitoring and Evaluation (DPME), Russia (vários stakeholders, incluindo entidades governamentais, universidades, ONGs, empresas de consultoria e a recentemente estabelecida associação nacional de avaliação), and The Joint United Nations Programme on HIVIAIDS (UNAIDS). Cada um desses casos usou um conjunto de competências para seus processos avaliativos.

A análise dos cinco casos ilustra diferentes usos de competências de avaliadores. Alguns usos são relacionados a processos de contratação / comissionamento para selecionar avaliadores que possam "[...] ajudá-los a tomar decisões sobre seus programas, políticas ou outras decisões gerenciais" (Podems, 2014, p. 132). Outro tipo de uso está focado no processo de aprendizagem de uma avaliação, incluindo o desenho de programas de treinamento, ensino, e orientação a avaliadores jovens e emergentes. A lista de competências poderia também ser usada por praticantes como uma ferramenta de auto avaliação para melhorar seus conhecimentos e habilidades. Finalmente, no caso da ANZEA, competências foram usadas para simplesmente ajudar a comunidade de avaliadores em suas necessidades de aprendizado e desenvolvimento em geral. Os cinco casos também revelam que as diferentes fases do processo de construção de competências não foram conduzidas pelos mesmos grupos de atores, uma vez que alguns casos levaram muitos anos entre o início e o final. Os casos mencionam a importância do engajamento de vários atores para desenvolver uma lista de competências.

Outras iniciativas, mencionadas pelo UNEG (2016), são os programas voluntários de revisão entre pares, similares ao modelo conduzidos pela Sociedade Europeia de Avaliação (EES) em parceria com a Sociedade de Avaliação do Reino Unido (UKES). Esse modelo foi criado para ajudar avaliadores, que exercem diferentes papeis, a identificar seus pontos fracos e desenvolver soluções para superá-los. Ele combina autorreflexão guiada e diálogo com dois revisores de pares, membros seniores da EES (2020a), via e-mail ou videoconferência.

A análise dos cinco casos e a iniciativa da EES/UKES mostram que a definição de um conjunto de competências e capacidades de avaliadores podem ter muitos usos além de discriminar quem pode ou não ser considerado avaliador.

Reconhecimento do conhecimento, habilidades e experiências

Uma outra vertente da profissionalização é o reconhecimento dos conhecimentos, habilidades e experiências, a partir de um corpo de conhecimentos amplamente reconhecidos, formado a partir do desenvolvimento da teoria, pesquisas e formação de alto nível. Ao mesmo tempo em que a consciência da importância da avaliação vem crescendo constantemente, ainda existe muito ceticismo sobre a qualidade dos profissionais que realizam avaliações e sobre 
seus resultados (Rodriguez-Bilella, 2017). Assim, os avaliadores ainda têm que avançar para serem reconhecidos pelo público, ou pelo mercado, como possuidores de conhecimentos e competências especiais e preparados para aplicar esses conhecimentos e competências no interesse dos outros.

O julgamento preciso do conhecimento, das habilidades e experiências dos profissionais é necessário para selecionar professores, pesquisadores, especialistas ou equipes de consultores em avaliação. Geralmente é baseado na análise de currículos, portfólios, declarações, entrevistas e propostas. No entanto, algumas VOPEs avançaram em mecanismos de reconhecimento mais formais. É o caso da Sociedade Canadense de Avaliação (CES), que lançou o Programa de Credenciamento de Avaliadores (PCA) em junho de 2009. O objetivo do programa é promover avaliação ética, de alta qualidade e competente no Canadá. O Programa foi fundado em três pilares: padrões de avaliação, competências do avaliador e um código de ética.

Lahey et al. (2018) estudaram a natureza do mercado de avaliação canadense, do lado da "oferta" e do lado da "demanda". Eles concluíram que a designação de avaliadores credenciados canadenses pode impactar o mercado de avaliação no futuro por meio de um aumento na qualidade das avaliações, um melhor equilíbrio de preços para serviços em oposição a prestadores de serviços que oferecem preços muito baixos, proporcionando estabilidade no serviço por parte de fornecedores. Também pode melhorar o entendimento da avaliação pela demanda em geral e aumentar o interesse na contratação de serviços de avaliação.

Outro estudo entrevistou 654 membros da CES em abril de 2014 sobre questões de profissionalização e o PCA. Os resultados revelaram que, em geral, as atitudes e percepções em relação ao PCA foram positivas. Os membros que participaram do programa e foram credenciados tenderam a relacionar o programa a melhorias em sua prática e a um maior sentimento de pertencimento à comunidade de avaliação (Gauthier et al., 2015).

Em 2015, uma avaliação formativa do PCA foi conduzida pelo Centro de Avaliação de Claremont em relação à sua eficácia, relevância / utilidade, eficiência, impactos indesejados e sustentabilidade. O estudo pesquisou 706 membros da CES, 336 não membros e entrevistou líderes da CES, membros do Conselho de diretores da CES e membros do conselho de credenciamento, contratantes de avaliação canadenses, empregadores de avaliadores no Canadá, parceiros em potencial PCA e indivíduos que criticaram o programa no passado. A CES foi pioneira na implementação de um processo de credenciamento de avaliadores e o programa tinha apenas 6 anos quando a avaliação foi realizada. Os resultados mostraram que menos da metade dos avaliadores credenciados apresentou melhora na comercialização ou no alcance de seus objetivos de carreira. Os altos custos e tempo associados ao processo representam uma barreira entre os avaliadores que não buscaram o credenciamento. Do lado dos contratantes, não se verifica um forte grau de aceitação e apoio (Fierro et al., 2016).

\section{Estruturas institucionais}

Estruturas institucionais podem ser elementos essenciais para promover um ambiente favorável ao desenvolvimento ou fortalecimento da cultura, do conhecimento e da prática do campo da avaliação. Essas estruturas podem ser governamentais, consideradas essenciais para promover a prática e o uso das avaliações para melhoria de políticas públicas. Alguns países avançaram no sentido de ter conselhos ou políticas nacionais de avaliação, como o México. Podem também ser iniciativas não governamentais, a exemplo das VOPEs. Segundo Rodriguez-Bilella \& Lucero (2016), as VOPEs nacionais e regionais tendem a oferecer uma vasta gama de atividades, como: disseminação de conhecimentos advindos da prática do campo, disseminação da cultura avaliativa, desenvolvimento de capacidades e habilidades, o que se dá geralmente por meio seminários e workshops; desenvolvimento de padrões e princípios para guiar avaliações, e desenvolvimento de capacidades e competências, entre outras. Para Rodriguez-Bilella \& Lucero (2016, p. 48), esse conjunto de atividades expressa os “[...] principais princípios das VOPEs, de que avaliação é uma prática que pode ser fortalecida pelo esforço profissional e coletivo de pares trabalhando juntos de maneira organizada". 
A criação da primeira associação de profissionais sobre o tema, a Sociedade Canadense de Avaliação (CES, 2020), em 1981, seguida da Associação Americana de Avaliação (AEA)', em 1986, abriu o caminho para a formação de outras VOPEs. Segundo o Rugh et al. (2013) o número de VOPEs, nacionais e transnacionais, cresceu de 15, na década de 90, para mais de 155, em 2013, conforme minucioso levantamento feito pela Organização Internacional de Cooperação em Avaliação (IOCE, na sigla em inglês). No Brasil, a RBMA foi criada em 2008, como iniciativa informal, e institucionalizada em 2014 como uma associação sem fins lucrativos.

Além de redes e associações nacionais e regionais independentes, existem as instituições ligadas a organizações de cooperação multilateral, como o Comitê de Assistência ao Desenvolvimento da Organização para a Cooperação e o Desenvolvimento Econômico (CAD/ OCDE), e o Grupo de Avaliação da Organização das Nações Unidas (UNEG, na sigla em inglês) que têm promovido diversas iniciativas para o desenvolvimento de capacidades nacionais, institucionais e individuais.

\section{As diretrizes para a prática de avaliação no Brasil}

As diretrizes brasileiras (Silva et al., 2020), lançadas pela RBMA, em setembro de 2020, chegam num momento de múltiplas crises. A crise sanitária causada pela pandemia COVID-19, por exemplo, tem se valido de técnicas de análises de dados, e de pesquisas visando subsidiar a sociedade e a comunidade científica com informações relevantes para a tomada de decisão em diversas instâncias e níveis. Nunca antes a mídia usou tantos dados e indicadores. As outras crises, com menos intensidade, também se valem de estudos e pesquisas para guiarem suas decisões. Fica cada vez mais evidente, para a sociedade como um todo, a importância de se ter evidências críveis para tomar decisões e para disseminar informações confiáveis. Em última instância, e conforme o livro das diretrizes argumenta, a avaliação está fortemente conectada com a democracia e com a urgente redução das desigualdades e suas diversas manifestações no Brasil. Por isso é cada vez mais fundamental impulsionar a avaliação no Brasil enquanto campo profissional, que deve rapidamente se desenvolver, se modernizar e amadurecer.

Segundo Silva et al. (2020), o sentido de se produzir as diretrizes brasileiras é convocar a comunidade brasileira e a própria sociedade a ampliar sua atenção e suas capacidades para influenciar a produção de avaliações, questionar a forma como têm sido realizadas e seus propósitos, bem como realizá-las e usá-las da melhor forma possível. Já foram mencionados, nesse ensaio, os fatores que levam à percepção do quanto o campo da avaliação vem se desenvolvendo no Brasil nas duas últimas décadas. Seguindo os pilares da profissionalização, este seria um caminho natural para que a comunidade brasileira construísse suas próprias diretrizes. Graças à sua capacidade de convocação, construída desde a criação, em 2008, e ao apoio de parceiros importantes como a Agenda de Avaliação do GIFE, a RBMA coordenou o processo de elaboração das diretrizes, que envolveu os principais atores da comunidade avaliativa brasileira.

Conforme descrito nas Diretrizes para a Prática de Avaliação no Brasil, o processo de construção se deu em diversas etapas. Primeiramente, vinte especialistas brasileiros com notório saber e experiência prática em avaliação foram consultados sobre a versão traduzida dos Estandares da ReLAC. A análise desses 20 nomes e suas filiações institucionais revela que houve uma preocupação na diversidade em termos de gênero, das temáticas em que atuam, e do seu lugar de fala. Dos especialistas entrevistados, 50\% eram mulheres e 50\% homens; $40 \%$ eram ligados à temática do desenvolvimento social, $20 \%$ à saúde, $15 \%$ à educação, $10 \%$ a políticas públicas e $15 \%$ outros temas; $50 \%$ tinham como lugar de fala a academia e centros de pesquisa, $20 \%$ consultores em avaliação, $20 \%$ investidores e contratantes e $10 \%$ representantes de governos. As consultas feitas aos especialistas geraram uma segunda versão do documento.

${ }^{1}$ Que surgiu a partir da fusão da Evaluation Network (ENet) e da Evaluation Research Society (ERS). Fonte: AEA (2020b). 
A segunda versão foi submetida à análise de um grupo mais abrangente, profissionais envolvidos ou interessados pelo tema da avaliação, por intermédio de uma pesquisa online. Das 200 pessoas convidadas a participar, 105 responderam ao questionário:

As 105 respostas recebidas vieram de um grupo com $50 \%$ de residentes na região Sudeste, $61 \%$ de mulheres, $61 \%$ acima de 45 anos e com $64 \%$ autodeclarados brancos. $37 \%$ deles afirmaram possuir mestrado e $45 \%$ doutorado. $72 \%$ dos respondentes atuam com pesquisa, consultoria e docência. Os números não possuem validade estatística para a comunidade geral de interessados em avaliação no Brasil, mas demonstram a evidente familiaridade com o tema da avaliação por parte dos respondentes. (Silva et al., 2020, p. 37).

A partir da incorporação das contribuições dos 105 profissionais, uma terceira versão foi produzida. Uma terceira etapa de consulta à comunidade de avaliadores gerou uma série de rodas virtuais de conversa. Silva et al. $(2020$, p.43) relatam que

[...] os grupos consultados foram os jovens avaliadores da RBMA, profissionais de organizações da sociedade civil rotineiramente financiadas por fundações, institutos, empresas e programas de fomento, pessoas negras que trabalham com pesquisa e avaliação, além de duas rodas com diversos interessados em avaliação das regiões SUL/CO/SE e N/NE.

Participaram dessas rodas virtuais de discussão 62 pessoas. Esses elementos colhidos geraram mais uma revisão no documento, que chegou à sua quarta versão.

A última etapa foi formada por duas consultas. A primeira foi feita ao grupo da Agenda de Avaliação do GIFE, formado por representantes de 4 investidores sociais privados e a segunda, ao grupo de conselheiros e diretores da RBMA. Essas últimas contribuições foram incorporadas ao documento e resultaram na versão final.

As diretrizes brasileiras incluem as seguintes dimensões: aprendizagem e utilização, com 7 diretrizes, direitos e integridades, também com 7 diretrizes, contextualização e valorização, com 4 diretrizes e método e viabilidade, com 8 diretrizes, totalizando 26 diretrizes. 0 livro eletrônico pode ser acessado na biblioteca virtual do GIFE (Silva et al., 2020).

À luz dos fatores que influenciam a profissionalização da avaliação propostos pela UNEG (2016) e discutidos neste ensaio, as diretrizes brasileiras são uma peça chave para o caminho da profissionalização no país. Potencialmente, podem provocar reflexão sobre o conteúdo e abordagem dos diversos programas de formação e seus resultados de aprendizagem para aqueles que serão os futuros especialistas, pesquisadores e professores desse campo. Podem também orientar agentes públicos e privados no desenho de avaliações, em seus processos de contratação de serviços especializados, quando for o caso, e no uso e apropriação dos resultados das avaliações. Pensando nas avaliações como instrumento de fortalecimento da democracia, as diretrizes podem ainda ajudar conselheiros de políticas e jornalistas em seu papel de supervisionar e dar transparência a políticas e serviços públicos.

As diretrizes abrem também caminho para discussões futuras a respeito de competências e capacidades dos avaliadores e até do papel da RBMA como agente articulador e influenciador da profissionalização deste campo no Brasil.

\section{Conclusão}

De acordo com a estrutura proposta pelo UNEG (2016), seis fatores podem contribuir para a profissionalização nos diversos países: acesso à educação, formação e treinamento; disseminação do conhecimento e boas práticas; diretrizes, princípios éticos e padrões para a prática da avaliação; um conjunto de competências e capacidades, reconhecimento do conhecimento, habilidades e experiências dos profissionais e estruturas institucionais.

O acesso à educação é fator essencial para a preparação de profissionais que exercem ou exercerão atividades relacionadas ao campo da avaliação. A oferta de programas de formação 
nessa área é bastante significativa nos Estados Unidos, na Europa, no Canada e tem crescido sobremaneira na América Latina e no Brasil. No entanto, a falta de processos de acreditação específicos nesse campo não permite afirmar se a oferta de programas é suficiente ou adequada para que esses profissionais produzam avaliações ou pesquisas de qualidade, de acordo com as demandas do mercado.

A disseminação de conhecimentos e boas práticas tem forte influência para a profissionalização do campo, pelo fato de avaliações serem, em geral, estudos empíricos. Os teóricos e especialistas que têm publicado considerável volume de artigos e livros e as VOPEs, por meio de revistas, conferências e workshops, têm sido os principais agentes de disseminação de conhecimento no campo da avaliação, construindo verdadeiras comunidades de aprendizagem no mundo todo.

Diretrizes, princípios éticos e padrões são a base de diversas profissões. No campo da avaliação diversas VOPEs, inclusive a RBMA, e outras organizações internacionais como UNEG e CAD/OCDE têm elaborado diretrizes para a prática da avaliação. As diretrizes podem ter grande importância em apoio aos processos de treinamento e formação de profissionais, por exemplo, os relacionados à prática avaliativa e seus desafios éticos, que podem ser elucidados por casos práticos (Bustelo, 2006). Também podem ser usadas em processos de credenciamento e fomentar um sentimento de pertencimento e identidade entre os profissionais das diversas comunidades de avaliadores nos países, fortalecendo uma cultura de avaliação.

A definição de um conjunto de capacidades e competências pode nortear a prática avaliativa em diversos aspectos, servindo como base, por exemplo, para programas de treinamento e em processos de certificação ou credenciamento. Pesquisa multinacional composta por cinco estudos de caso conduzida por Podems (2014) mapeou diferentes tipos de uso de capacidades e competências, identificando as seguintes possibilidades: basear processos de contratação e seleção de avaliadores; servir como instrumento de auto avaliação; ou simplesmente para ajudar a comunidade de avaliadores em suas necessidades de aprendizado e desenvolvimento em geral. Esses e outros exemplos mostram que competências e capacidades podem ter muitos usos além de discriminar quem pode ou não ser considerado avaliador.

A definição de critérios ou processos para o reconhecimento do conhecimento, habilidades e experiências de profissionais no campo da avaliação é importante para dar aos contratantes de estudos avaliativos ou de profissionais a segurança de que estão fazendo as escolhas corretas, reduzindo os riscos de trabalhos de baixa qualidade. Embora a Sociedade Canadense de Avaliação tenha implementado um programa de credenciamento de avaliadores, ainda enfrenta desafios como resistência dos profissionais ao custo e tempo relacionado ao processo e a falta de apoio consistente dos contratantes.

As estruturas institucionais, governamentais ou não, têm um papel importante para a promoção de um ambiente favorável ao desenvolvimento e para o fortalecimento da cultura avaliativa nos países, bem como para a disseminação de conhecimento teórico e empírico, desenvolvimento de capacidades e habilidades, desenvolvimento de diretrizes, princípios e padrões e de capacidades e competências. Tais estruturas são, portanto, articuladoras dos demais fatores influenciadores da profissionalização da avaliação. As VOPEs são o tipo de organização mais atuantes nos países, conforme Rodriguez-Bilella \& Lucero (2016).

As diretrizes para a Prática de Avaliação no Brasil são um passo importante para o caminho da profissionalização da avaliação no Brasil, especialmente porque nascem com um sentimento de pertencimento e apropriação da comunidade avaliativa brasileira, pela maneira participativa com que foram construídas. Junto com as crescentes oportunidades de formação, a atuação da RBMA, hoje com mais de 8.500 seguidores, e os diversos canais para a disseminação de conhecimento, uma comunidade crescente de profissionais vem se formando e debatendo o real valor que a avaliação pode agregar ao desenvolvimento do país e à nossa democracia. Como toda novidade, esse documento precisa ser testado pela própria comunidade, seu uso escrutinado por meio de pesquisas e revisões periódicas. Para que isso aconteça, é preciso disseminá-lo, dialogar sobre ele, fazê-lo conhecido. Isso pode trazer um novo patamar de debates e pesquisas no campo brasileiro: o olhar para a prática da avaliação, a quem tem 
servido, com que propósitos, com que tipo de abordagens e métodos, ouvindo que tipo de atores. Esse olhar faz parte do aprendizado que vem da reflexão.

Há outros passos igualmente importantes a serem trilhados. A observação do desenvolvimento do campo em outras partes do mundo e a reflexão sobre a estrutura proposta pelo UNEG, podem motivar organizações como a RBMA a formular estratégias que conduzirão a prática da avaliação no Brasil a um patamar maior de credibilidade e qualidade.

\section{Fonte de financiamento}

Coordenação de Aperfeiçoamento de Programas de Estudos Superiores (CAPES).

\section{Conflito de interesse}

Não há.

\section{Referências}

American Evaluation Association - AEA. (2020a). Competencies. Recuperado em 9 de dezembro de 2020 de https://www.eval.org/page/competencies

American Evaluation Association - AEA. (2020b). Guiding principles for evaluators. Recuperado em 9 de dezembro de 2020, de https://www.eval.org/p/cm/ld/fid=51

American Evaluation Association - AEA. (2020c). University programs. Recuperado em 20 de dezembro de 2020, de https://www.eval.org/p/cm/ld/fid=43

American Evaluation Association - AEA. (2020d). Recuperado em 20 de dezembro de 2020, de https:// www.eval.org/p/cm/ld/fid=472

Australian Council of Professions - ACoP. (2021). What is a profession? Professions professionals professionalism. Recuperado em 26 de janeiro de 2021, de https://www.professions.org.au/what-is-aprofessional/

Brasil. Ministério do Desenvolvimento Social e Combate a Fome - MDS. Secretaria de Avaliação e Gestão da Informação. (2016). SAGI: Informação e conhecimento para políticas de desenvolvimento social. Brasília.

Brasil. Ministério da Economia. (2020). Conselho de Monitoramento e Avaliação de Políticas Públicas (CMAP). Recuperado em 20 de dezembro de https://www.gov.br/economia/pt-br/acesso-a-informacao/ participacao-social/conselhos-e-orgaos-colegiados/cmap/institucional/institucional-1

Bustelo, María. (2006). The potential role of standards and guidelines in the development of an evaluation culture in Spain. Evaluation, 12(4), 437-453. http://dx.doi.org/10.1177/1356389006071291

Canadian Evaluation Society - CES. (2020). Recuperado em 20 de dezembro de https://evaluationcanada. ca/our-history\#: :text=The\%20CES\%20was\%20first\%20incorporated,field\%20of\%20evaluation\%20in\%20 Canada

Centro de Aprendizagem em Avaliação e Resultados para o Brasil e a África Lusófona - FGV EESP Clear. (2020). Cursos: Evidências no Ciclo da Política Pública. Recuperado 20 de dezembro de 2020, de http:// fgvclear.org/pt/cursos/

Consortium of Universities for Evaluation Education - CUEE. (2021). Evaluation education [L'enseignement de l'évaluation]. Recuperado 21 de janeiro de 2021, de https://evaluationeducation.ca/

Escola Nacional de Administração Pública - ENAP. (2021). Recuperado em 26 de janeiro de 2021, de https://www.enap.gov.br/pt/cursos/pos-graduacao/mestrado/mestrado-profissional-em-avaliacao-emonitoramento-de-politicas-publicas

European Evaluation Society - EES. (2020a). Recuperado 20 de dezembro de 2020, https:// europeanevaluation.org/voluntary-evaluator-peer-review/

European Evaluation Society - EES. (2020b). Education. Recuperado 20 de dezembro de 2020, de https:// europeanevaluation.org/education/

EvalPartners. (2018). Global Evaluation Agenda 2016-2020. Recuperado 9 de dezembro de 2020, de https:// www.evalpartners.org/global-evaluation-agenda

Faculdade Cesgranrio. (2020a). Revista Meta: Avaliação. Recuperado 20 de dezembro de 2020, de https:// revistas.cesgranrio.org.br/index.php/metaavaliacao

Faculdade Cesgranrio. (2020b). Ensaio: Avaliação e Políticas Públicas em Educação. Recuperado 20 de dezembro de 2020, de https://revistas.cesgranrio.org.br/index.php/ensaio

Faculdade Cesgranrio. (2020c). Gestão da avaliação. Recuperado em 20 de dezembro de 2020, de http:// facesg.edu.br/graduacao_avaliacao.aspx 
Faculdade Cesgranrio. (2021). Mestrado profissional em avaliação (stricto sensu). Recuperado em 26 de janeiro de 2021, de http://mestrado.cesgranrio.org.br/mestrado/selecao.aspx

Fierro, Leslie, Galport, Nicole, Hunt, Ashley, Codd, Heather, \& Donaldson, Stewart. (2016). Canadian Evaluation Society credentialed evaluator designation program: Evaluation report. Claremont: Claremont Evaluation Center. Recuperado 20 de dezembro de 2020, de https://evaluationcanada.ca/txt/2016_pdp_ evalrep_en.pdf

Fundação Carlos Chagas - FCC. (2020). Estudos em Avaliação Educacional. Recuperado 20 de dezembro de 2020, de http://publicacoes.fcc.org.br/ojs/index.php/eae

Fundação Getúlio Vargas - FGV. (2020). Curso de formação em monitoramento e avaliação. Recuperado em 20 de dezembro de 2020, de http://fgvclear.org/site/wp-content/uploads/folder_forma-atualizado-1.pdf Fundação Itaú Social. (2021). Recuperado em 26 de janeiro de 2021, de https://www.redeitausocialdeavaliacao. org.br/

Fundação João Pinheiro - FJP. (2020, janeiro 14). Curso de Especialização em Monitoramento e Avaliação de Políticas Públicas. Recuperado em 26 de janeiro de 2021, de http://novosite.fjp.mg.gov.br/especializacaoem-monitoramento-e-avaliacao-de-politicas-publicas/

Gauthier, Benoît, Kishchuk, Natalie, Borys, Shelley, \& Roy, Simon. (2015). The CES professional designations program: Views from members. The Canadian Journal of Program Evaluation, 29(3), 98-133. http://dx.doi. org/10.3138/cjpe.29.3.98

Gullickson, Amy, King, Jean, LaVelle, John, \& Clinton, Janet. (2019). The current state of evaluator education: A situation analysis and call to action. Evaluation and Program Planning, 75, 20-30. PMid:31015094. http:// dx.doi.org/10.1016/j.evalprogplan.2019.02.012

Lahey, Robert, Elliott, Catherine, \& Heath, Sarah. (2018). The evolving market for systematic evaluation in Canada. New Directions for Evaluation, 2018(160), 45-62. http://dx.doi.org/10.1002/ev.20346

Mertens, Donna. (2008). Transformative research and evaluation. New York: Guilford Press.

Meyer, Wolfgang. (2016). Toward professionalization? The contribution of university-based training programs in pioneer countries. In Reinhard Stockmann \& Wolfgang Meyer (Eds.), The future of evaluation (pp. 98-112). London: Palgrave Macmillan. http://dx.doi.org/10.1057/9781137376374_7

Morris, Michael. (2011). The good, the bad, and the evaluator: 25 years of AJE ethics. The American Journal of Evaluation, 32(1), 134-151. http://dx.doi.org/10.1177/1098214010388267

Organização para a Cooperação e Desenvolvimento Econômico - OCDE. Comitê de Ajuda ao Desenvolvimento - CAD. (2010). Normas de qualidade para a avaliação do desenvolvimento. Paris: CAD/OCDE.

Patton, Michael Quinn. (2008). Utilization-focused evaluation. Thousand Oaks: SAGE Publications.

Podems, Donna. (2014). Evaluator competencies and professionalizing the field: Where are we now? The Canadian Journal of Program Evaluation, 28(3), 10.

Podems, Donna. (2018). Being an evaluator: Your practical guide to evaluation. New York: Guilford Publications.

Red Latinoamericana de Seguimiento, Evaluación y Sistematización - ReLAC. (2016). Estándares de evaluación para América Latina y el Caribe. Recuperado 20 de dezembro de 2020, de https://www.relac. net/biblioteca/evaluation-standards/

Rede Brasileira de Monitoramento e Avaliação - RBMA. (2021a). Recuperado em 26 de janeiro de 2021, de http://redebrasileirademea.ning.com

Rede Brasileira de Monitoramento e Avaliação - RBMA. (2021b). Revista Brasileira de Avaliação (RBAVAL). Recuperado em 26 de janeiro de 2021, de https://www.rbaval.org.br/

Rodriguez-Bilella, Pablo. (2017). Significance of voluntary organizations for professional evaluation (VOPES) for the dissemination and professionalization of evaluation. Zeitschrift für Evaluation, 16, 210-218.

Rodriguez-Bilella, Pablo. (2018). Más de cuarenta posgrados en evaluación en América Latina y el Caribe: Al Borde del Caos. Recuperado 20 de dezembro de 2020, de https://albordedelcaos.com/2018/05/17/masde-cuarenta-posgrados-en-evaluacion-en-america-latina-y-el-caribe/

Rodriguez-Bilella, Pablo, \& Lucero, María Alejandra. (2016). Evaluation as a global phenomenon: The development of transnational networks. In Reinhard Stockmann \& Wolfgang Meyer (Eds.), The future of evaluation: Global trends, new challenges, shared perspectives (pp. 66-80). London: Palgrave MacMillan. http://dx.doi.org/10.1057/9781137376374_5.

Rugh, J., Akundy, A., \& Segone, M. (2013). Voluntary Organizations for Professional Evaluation (VOPEs): Learning from Africa, Americas, Asia, Australasia, Europe and Middle East. Unicef. Recuperado em 26 de janeiro de 2021 https://www.evalpartners.org/sites/default/files/UNICEF_NY_ECS_Book2_web.pdf

Schwandt, Thomas. (2015). Evaluation foundations revisited: Cultivating a life of the mind for practice. Stanford: Stanford University Press. http://dx.doi.org/10.1515/9780804795722.

Scientific Electronic Library Online - SciELO. (2020). Avaliação: Revista da Avaliação da Educação Superior. Recuperado 20 de dezembro de 2020, de https://submission.scielo.br/index.php/aval

Silva, Rogério, Joppert, Marcia, \& Gasparini, Max. (2020). Diretrizes para a prática de avaliação no Brasil (1. ed.). Rio de Janeiro: Rede Brasileira de Monitoramento e Avaliação. Recuperado 20 de dezembro de 2020 de https://sinapse.gife.org.br/download/diretrizes-para-pratica-de-avaliacoes-no-brasil 
Stevahn, Laurie, King, Jean, Ghere, Gail, \& Minnema, Jane. (2005). Establishing essential competencies for program evaluators. The American Journal of Evaluation, 26(1), 43-59. http://dx.doi. org/10.1177/1098214004273180

Stockmann, R. (2016). The future of evaluation in modern societies. In R. Stockmann \& W. Meyer (Eds.), The Future of Evaluation: Global Trends, New Challenges, Shared Perspectives (pp. 36-50). Palgrave Macmillan UK. https://doi.org/10.1057/9781137376374_3

Stufflebeam, Daniel, \& Coryn, Chris. (2014). Evaluation theory, models, and applications. New York: John Wiley \& Sons.

The United Nations Evaluation Group - UNEG. (2013). Core competencies for evaluators of the UN system. New York.

The United Nations Evaluation Group - UNEG. (2016). Professionalization of evaluation concept paper. New York.

The United Nations Evaluation Group - UNEG. (2017). Norms and standards for evaluation. New York.

Universidade Federal de Juiz de Fora - UFJF. (2021). Mestrado em Gestão e Avaliação da Educação Pública: Programa de Pós-graduação Profissional. Recuperado em 26 de janeiro de 2021, de http://mestrado. caedufjf.net/

Universidade Federal de Santa Catarina - UFSC. (2021). Programa de Pós-graduação em Métodos e Gestão em Avaliação. Recuperado em 26 de janeiro de 2021, de https://ppgmga.posgrad.ufsc.br/

Universidade Federal do Ceará - UFC. (2020). Revista Avaliação de Políticas Públicas (AVAL). Recuperado 20 de dezembro de 2020, de http://www.avalrevista.ufc.br/index.php/revistaaval

Universidade Federal do Ceará - UFC. (2021). Programa de Pós-graduação em Avaliação de Políticas Públicas. Recuperado em 26 de janeiro de 2021, de https://ppgapp.ufc.br/pt/

Wilcox, Yuanjing, \& King, Jean A. (2012). A professional grounding and history of the development and formal use of evaluator competencies. The Canadian Journal of Program Evaluation, 3(28), 1-28. 